\title{
Comparison of Cadmium-Zinc-Telluride semiconductor and Yttrium-Aluminum-Perovskite scintillator as photon detectors for epithermal neutron spectroscopy
}

\author{
M. Tardocchi ${ }^{\mathrm{a}, *}$, A. Pietropaolo ${ }^{\mathrm{b}}$, C. Andreani ${ }^{\mathrm{b}}$, G. Gorini $^{\mathrm{a}}, \mathrm{S}$. Imberti ${ }^{\mathrm{b}}$, E. Perelli-Cippo $^{\mathrm{a}}$, \\ R. Senesi ${ }^{\mathrm{b}}$, N. Rhodes ${ }^{\mathrm{c}}$, E.M. Schooneveld ${ }^{\mathrm{c}}$ \\ ${ }^{a}$ CNR-INFM and Dipartimento di Fisica "G. Occhialini”, Università degli Studi di Milano-Bicocca, Piazza Della Scienza 3, I-20126 Milano, Italy \\ ${ }^{\mathrm{b}}$ CNR-INFM and Dipartimento di Fisica, Università degli Studi di Roma "Tor Vergata", Roma, Italy
}

${ }^{\mathrm{c}}$ ISIS Facility, Rutherford Appleton Laboratory, Chilton, UK

\begin{abstract}
The range of applications of epithermal neutron scattering experiments has been recently extended by the development of the Resonance Detector. In a Resonance Detector, resonant neutron absorption in an analyzer foil results in prompt emission of X- and $\gamma$ rays which are detected by a photon counter. Several combinations of analyzer foils and photon detectors have been studied and tested over the years and best results have been obtained with the combination of a natural uranium and (i) Cadmium-Zinc-Telluride (CZT) semiconductor, (ii) Yttrium-Aluminum-Perovskite (YAP) scintillators. Here we compare the performance of the CZT semiconductor and YAP scintillator as Resonance Detector units.

Two Resonance Detector prototypes made of natural uranium foil viewed by CZT and YAP were tested on the VESUVIO spectrometer at the ISIS spallation neutron source. The results show that both YAP and CZT can be used to detect epithermal neutrons in the energy range from 1 up to $66 \mathrm{eV}$. It was found that the signal-to-background ratio of the measurement can significantly be improved by raising the lower level discrimination threshold on the $\gamma$ energy to about $600 \mathrm{keV}$. The advantages/disadvantages of the choice of a Resonance Detector based on YAP or CZT are discussed together with some potential applications.
\end{abstract}

(C) 2006 Elsevier B.V. All rights reserved.

PACS: 29.30.Hs; 29.30.Kv; 29.40.Wk; 61.12.Ex

Keywords: CZT; YAP; Epithermal neutron; Neutron spectroscopy; Resonance detector

\section{Introduction}

The recent developments of new instrumentation for epithermal neutron spectroscopy has been motivated by the extension of the kinematical region covered by today's neutron scattering experiments [1]. In particular, by accessing the kinematical region of both high and low wave vector transfers together with high energy transfers, new experimental studies on condensed matter systems become possible [1]. To perform neutron scattering experiments in this kinematical region the detection of

\footnotetext{
*Corresponding author. Tel.: + 390264482326 ; fax: + 390264482367

E-mail address: marco.tardocchi@mib.infn.it (M. Tardocchi).
}

epithermal neutrons in the $1-100 \mathrm{eV}$ energy range is required [2]. For this purpose the Resonance Detector (RD) has been developed for detection of epithermal neutrons on inverse geometry time of flight spectrometer operating at pulsed neutron sources [3].

Epithermal neutrons are routinely measured with the socalled Resonance Filter technique on the inverse geometry neutron spectrometer VESUVIO [4], installed at the ISIS pulsed neutron source. The Resonance Filter technique, which makes use of ${ }^{6} \mathrm{Li}$ glass scintillators for the detection of the scattered neutrons transmitted through an analyzer foil [5], has an intrinsic efficiency limited to neutron energies below $\sim 10 \mathrm{eV}$, due to the inverse square root energy dependence of the neutron capture cross-section of 
${ }^{6} \mathrm{Li}$. This limit has now been overtaken by the development of the RD technique. In the RD configuration neutron detection is divided in a two-step process (i) resonant neutron capture by an analyzer foil which strongly absorbs the scattered neutrons over a narrow energy interval; (ii) detection of the prompt radiative capture $\gamma$-ray cascade following the radiative decay of the compound nucleus to the ground state. The energy spectrum of the prompt $\gamma$ rays covers a wide range from tens of $\mathrm{keV}$ up to few $\mathrm{MeV}$ values of the neutron binding energy in the absorbing nucleus. The role of the photon detector is, by detecting the photon cascade, to tag the arrival time of the absorbed neutron. The full kinematics of the scattering event (wave vector and energy transfers) is reconstructed through the time-of-flight technique.

Several combinations of analyzer foils and photon detectors have been studied and tested on VESUVIO as RD [6]. The best results have been obtained with the combination of natural uranium or gold foils and (i) Cadmium-Zinc-Telluride (CZT) semiconductor, (ii) Yttrium-Aluminum-Perovskite (YAP) scintillators [7-9]. In this work the performance of the CZT semiconductor and YAP scintillator as RD units are compared. Advantages/ disadvantages of the choice of a RD based on YAP or CZT are discussed together with some potential applications.

\section{RD prototypes based on CZT and YAP}

The development work on the RD has been performed on the VESUVIO neutron spectrometer [4] at the ISIS spallation neutron sources. On VESUVIO (see Fig. 1) the neutron beam produced by the spallation source is undermoderated in water and collimated into the scattering sample, located inside an aluminum vacuum chamber. Scattered neutrons were detected by two RD prototype units which were positioned in the forward direction, at a close distance from the sample $(\approx 40 \mathrm{~cm})$ to increase the count rate. Two RD prototype units were made of a CZT semiconductor and a YAP scintillator viewing natural uranium analyser foils of $60 \mu \mathrm{m}$ thickness; both detectors were used without any shielding.

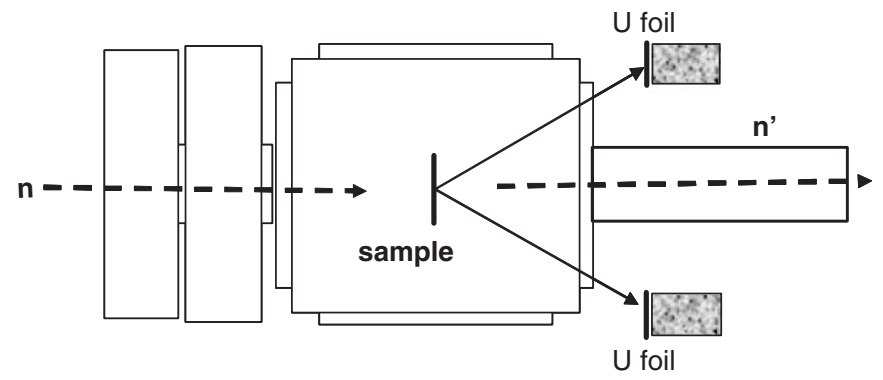

The CZT semiconductor detector $\left(5 \times 5 \times 5 \mathrm{~mm}^{3}\right)$ was a commercial product from eVPRODUCTS with an anodized aluminum housing [8]. The energy resolution was $4 \mathrm{keV}$ at $122 \mathrm{keV}$ with a peak-to-valley ratio of about 7 [8]. The YAP crystal (cylindrical shape of $35 \mathrm{~mm}$ diameter by $6.4 \mathrm{~mm}$ thickness) was manufactured by Bicron and was directly glued on a standard PM-tube with bialkali photocathode [9].

The two detectors were chosen with the requirement of having similar efficiency to detect prompt radiative capture $\gamma$-rays from ${ }^{238} \mathrm{U}$, which has the highest $\gamma$ line at $4.060 \mathrm{MeV}$. In Fig. 2 the computed total absorption probability for the two crystals is plotted in the $10-10^{4} \mathrm{keV}$ energy range [10]. Below $70 \mathrm{keV}$ photoelectric interaction is the dominant mechanism for energy deposition into the crystals providing $100 \%$ probability for both detectors. Above $200 \mathrm{keV}$ Compton interaction becomes dominant and guarantees a significant interaction probability up to $10 \mathrm{MeV}$ where it flattens at the $10 \%$ level. The CZT detector shows a higher efficiency than YAP between 70 and $400 \mathrm{keV}$, while the contrary happens between $400 \mathrm{keV}$ and $7 \mathrm{MeV}$. These two contributions compensate each other to give an overall interaction probability integrated over the entire energy range which is the same for the two detectors, the difference being less than $2 \%$.

A comparison of the pulse height response of the two detectors to $\gamma$-rays was done with a ${ }^{60} \mathrm{Co}$ radioactive source $(\gamma$ lines at 1173 and $1332 \mathrm{keV})$. The two pulse height spectra, after suitable normalization (to the same collection time, distance from source and detector area), are shown in Fig. 3. Two considerations can be made: (i) CZT spectrum shows a better energy resolution than YAP, as expected (ii) the measured integrated $\gamma$ flux is the same within the uncertainty in the normalization, confirming the interaction probability curve shown in Fig. 2.

The performances of the two RD prototypes were assessed with a Deep Inelastic Neutron Scattering [11] measurement on a reference polycrystalline lead sample. The results, shown in the form of time of flight spectra 
normalized to the detector area and beam current (in $\mu \mathrm{A} \mathrm{h}$, see Fig. 4), show peaks (signal) superimposed on a power law background. The peaks marked by dotted lines can be ascribed to $\mathrm{Pb}$ recoil signals corresponding to final neutron

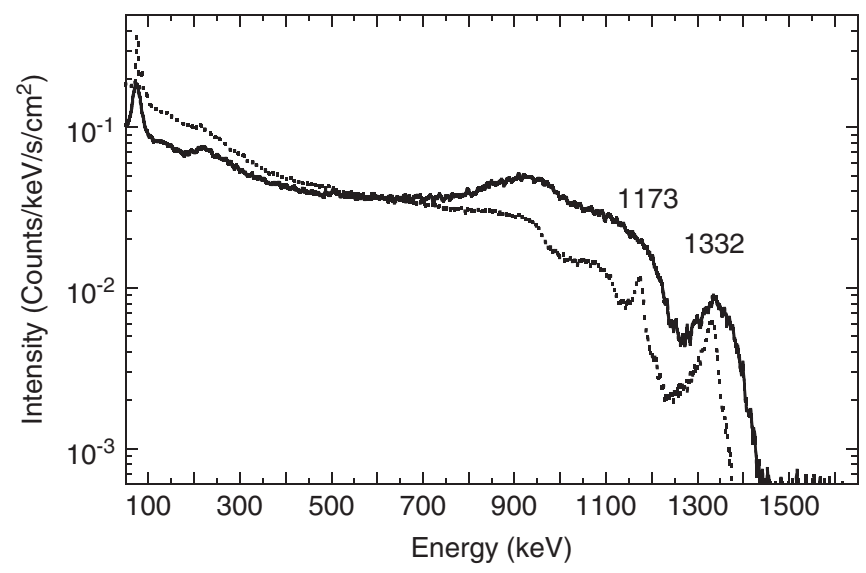

Fig. 3. Pulse height spectra recorded from a ${ }^{60} \mathrm{Co}$ source irradiating the CZT (continuous line) and YAP (dashed line) detectors. The full energy peaks are indicated with their corresponding energy in $\mathrm{keV}$ unit. energies of $6.67,20.9,36.7$ and $66.0 \mathrm{eV}$, respectively. The background has similar shapes for the two detectors, although the CZT data show few additional contamination peaks. The background is mainly due to neutron capture in the material surrounding the detectors, but there is also a significant fraction coming from the radioactive decay of ${ }^{238} \mathrm{U}[8,9]$. The data were collected with a low threshold on the $\gamma$ energy set at about 20 and $40 \mathrm{keV}$, for the CZT and YAP detector, respectively. Biparametric measurements [8] of the relative intensity of the photon pulse height spectra associated to each of the four neutron resonances, showed that the $\gamma$ emission spectrum is mostly independent on the absorbed neutron energy. In Ref. [8], it is also shown that a narrow energy selection improves the signal-to-background ratio but heavily depresses the signal intensity. This indicates that the poorer energy resolution of YAP with respect to CZT is not a limiting factor. On the other hand, it is shown in Ref. [9] that a significant improvement in the signal-to-background ratio can be achieved by raising the threshold at about $600 \mathrm{keV}$, due to a major $\gamma$-ray background source from boron (used in the shielding material) at about $500 \mathrm{keV}$. It was shown in Fig. 2 that for

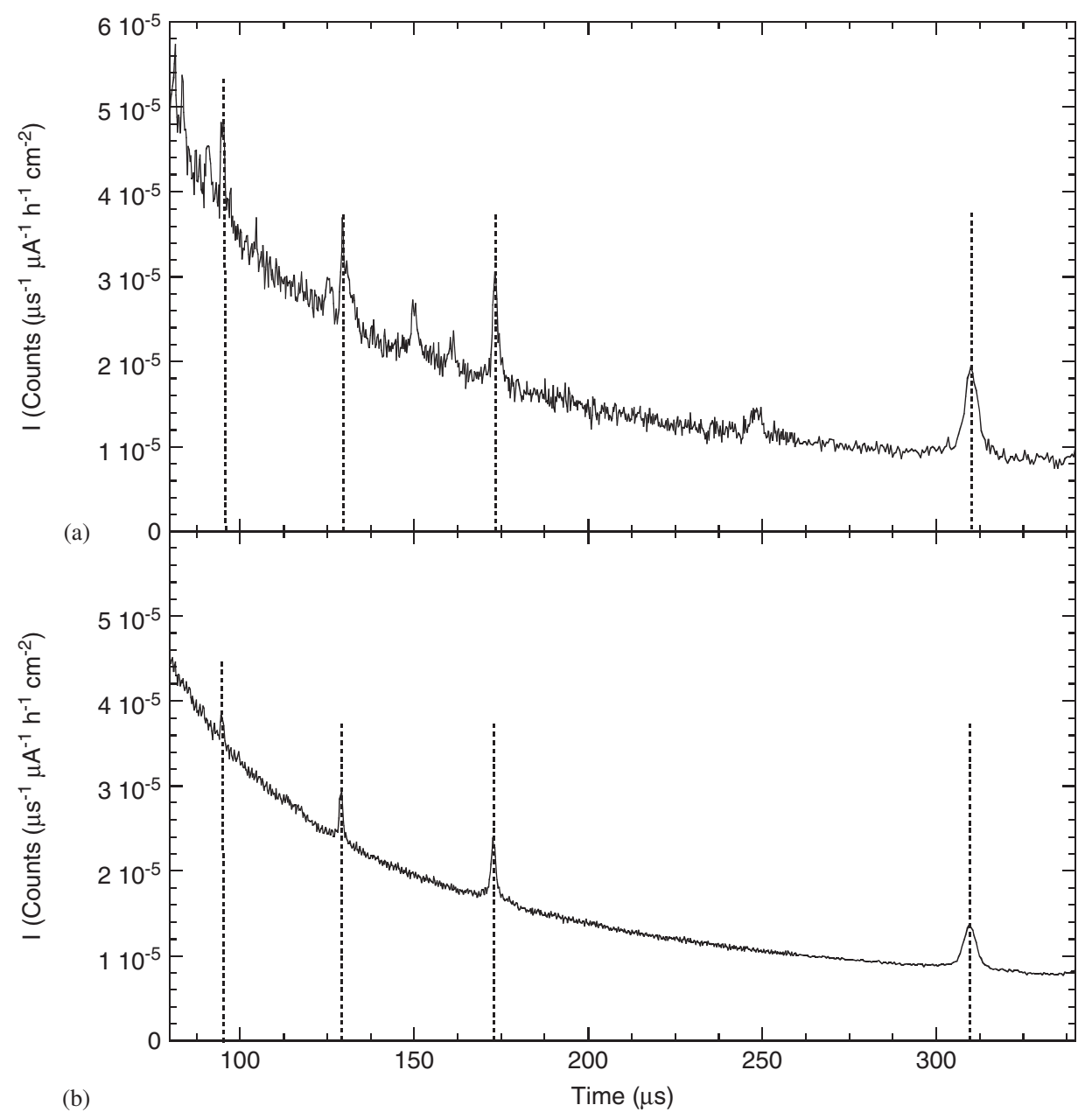

Fig. 4. Time of flight spectra in the time interval 80-340 $\mu$ s collected with the CZT (a) and YAP (b) detector from a Pb sample. The expected positions of four ${ }^{238} \mathrm{U}$ resonances (from 6.67 to $66.0 \mathrm{eV}$ from right to left) are indicated by the dashed vertical lines. 
$E_{\gamma}>600 \mathrm{keV}$ the YAP crystal has the advantage over CZT of a higher detection efficiency.

Some considerations should be made when going from a single RD unit to a position sensitive detector array. CZT has the disadvantage of being available in small crystals area. Pixelated CZT, which appeared in the market in the last years, could be used for detector arrays when good spatial resolution $(<5 \mathrm{~mm})$ is required. One unique property of the CZT is that it has been shown to have the dual function of detector for both epithermal and thermal neutrons, the latter due to the sensitivity of $\mathrm{Cd}$ to neutrons [7]. YAP on the other hand is insensitive to neutrons, can be machined to many different shapes and it offers a lower cost per detector area. RD based on YAP can thus be used to cover large detector areas.

\section{Applications of the resonance detector}

The first application of the RD is the upgrade of the VESUVIO to enhance its performance for epithermal neutron spectroscopy measurements. A new detector bank which covers the angular scattering regions from $40^{\circ}$ to $60^{\circ}$ has been built based on $32 \mathrm{RD}$ units made of YAP and gold foils. The first measurements with the new RD bank on a reference lead sample have indicated that the performance exceeds in terms of detection efficiency the ${ }^{6} \mathrm{Li}$ glass scintillator bank even at $5 \mathrm{eV}$. The RD performance improvement gets larger with higher neutron energies.

A second application of the RD is the construction of the Very Low Angle Detector (VLAD) bank of the VESUVIO spectrometer which will cover the $1-5^{\circ}$ angular range. VLAD, which is made of YAP and natural uranium analyzer foils [12], is due for installation by the end of 2005 . Its unique capability of measuring simultaneously low wave vector transfers (obtainable by covering a small angular scattering angles) and high energy transfers $(>1 \mathrm{eV})$ will open up a new range of measurements called High Energy Inelastic Scattering [13].

\section{Conclusions}

The development of the RD on the VESUVIO spectrometer at ISIS has opened up new perspectives for epithermal neutron spectroscopy at spallation pulsed neutron sources. Two RD prototype units made of natural uranium analyzer foils viewed by a CZT semiconductor or a YAP scintillator have been compared. The results indicate that both RD prototypes can detect with similar efficiency epithermal neutrons in the energy range from 1 up to $66 \mathrm{eV}$. A special application of the RD is the VLAD which will allow new measurements in a neutron kinematical scattering region so far unexplored.

\section{References}

[1] Newport, et al., Nucl. Instr. and Meth. A 224 (1984) 120

[2] A. Pietropaolo, et al., Physica B 350 (2004) e857.

[3] G. Gorini, et al., Nucl. Instr. and Meth. A 529 (2004) 293.

[4] R. Senesi, et al., Physica B 276-278 (2000) 200.

[5] A.L. Fielding, et al., Nucl. Instr. and Meth. A 480 (2002) 680.

[6] C. Andreani, et al., Nucl. Instr. and Meth. A 481 (2002) 509; M. Tardocchi, et al., Physica B 350 (2004) e853.

[7] C. Andreani, et al., Appl. Phys. A 78 (2004) 903.

[8] M. Tardocchi, et al., Nucl. Instr. and Meth. A 526 (2004) 477.

[9] M. Tardocchi, et al., Rev. Sci. Instrum. 75 (2004) 4880.

[10] Data compiled by C. M. Rozsa, Bicron Brochure "Efficiency for selected scintillators", http://www.bicron.com.; Bob Redus, "Charge Trapping in XR-100T-CZT Detectors", Amptek Application Note ANCZT1 Rev. 1, 2000 and http://www.amptek.com/anczt1.html.

[11] S.W. Lovesey, Theory of Neutron Scattering from Condensed Matter, Oxford University Press, London, 1987.

[12] E. Perelli-Cippo, et al., IEEE Trans. Nucl. Sci. NS-52 (4) (2005) 1092.

[13] C. Andreani, et al., Appl. Phys. Lett. 85 (2004) 5454. 